\title{
Analysis of Security Issues in VolP
}

\author{
Akshay Chopra \\ Information Technology \\ Maharaja Surajmal Institute of \\ Technology \\ Delhi, India
}

\author{
Bhavya Chaudhary \\ Information Technology \\ Maharaja Surajmal Institute of \\ Technology \\ Delhi, India
}

\author{
Suman Mann \\ Reader, Department of \\ Information Technology \\ Maharaja Surajmal Institute of \\ Technology \\ Delhi, India
}

\begin{abstract}
The most common way of communication is obviously through voice exchange. There are mainly two ways by which voice can be easily transmitted over a network: PSTN (Public Switched Telephone Network) and VoIP (Voice over Internet Protocol). The VoIP is relatively new and is gaining more and more popularity as it offers a wide range of features and is much more cost effective as compared to the traditional PSTN. But the VoIP brings with it certain security threats which need to be resolved in order to make it a more reliable source of communication. One of the most concerning security threats of VoIP technology is VoIP Spoofing. Caller ID Spoofing is a technique in which a person takes the identity of a different person to make a call to a third party, thereby hiding his/her original identity. None of the existing protocols so far have been able to prevent Spoofing of calls. This research explains the fundamentals of the Session Initiation Protocol and the Asterisk Server. Also, a new system is proposed which makes use of Public Key Infrastructure which can be used to create digital certificates for the VoIP, thereby helping us to make VoIP calls which are more secure. The PKI is a form of Cryptography which is used to create and manage digital certificates. The PKI mainly consists of (i) a certificate authority that issues the certificates (ii) registration authority which helps in verifying the identity of users (iii) central directory to store the certificates. Also, this paper gives a brief overview of the Asterisk Server which is used to make calls over the Internet. The basic idea is to implement the SIP with the Asterisk Server and using the concepts of Public Key Infrastructure to provide the Asterisk Server with the desired Digital Certificates so as to authenticate the user which in turn will make calling over the Internet more secure.
\end{abstract}

\section{Keywords}

Voice over Internet Protocol, Session Initiation Protocol, VoIP Spoofing, Public Key Infrastructure, Asterisk Server, Digital Certificates

\section{INTRODUCTION}

Voice over Internet Protocol is a technology which allows user to make voice calls via the Internet Protocol. VoIP converts the analog sound signals into discrete packets and then sends it over the network. All we need to make a VoIP call is a microphone, speakers and an internet connection. The main advantages of using Internet to make calls is that (i) it is very cheap as compared to the traditional PSTN system of making calls (ii) offers a rich feature set and (iii) is highly flexible.

The term Internet Telephony refers to using the Internet to make a communication between two or more end points, rather than the Public Switched Telephone Network (PSTN).
In a conventional PSTN system, the calls are made through the circuit switching technique, in which each call occupies a dedicated network for the total duration of the call. No other user can use the same circuit unless it is released by the ongoing call. However, the packet switching technique is used in the case of Internet telephony. Here, the signals are converted into digital packets and sent over the network. The advantage of using the packet switching technique is that packets from multiple users/calls can use the network simultaneously. Due to various advantages of VoIP, it is an excellent prospect for the future. But with the existing weaknesses such as VoIP not being secure, it definitely cannot replace PSTN completely. Therefore, it becomes essential to devise new protocols and security mechanisms in order to make VoIP more secure and a much more reliable source of communication.

VoIP is mainly triggered by the Session Initiation Protocol (SIP). But, many examiners say that spoofing a VoIP call is reasonably easy. It is due to the nature of these calls that anyone using the appropriate software can easily spoof a Caller ID and thus can make illicit calls. Consequently, it becomes paramount to increase the security of the SIP. The existing techniques are not proficient enough to be able to detect the location of the originating call. It is crucial to avoid attacks rather that detecting where the attack is from.

\section{VOICE OVER INTERNET PROTOCOL}

As the name suggests, the basic function of VoIP is to transmit voice messages over the internet. This technology enables the transmission of voice in any IP Digital Network. As suggested earlier, it makes use of packet-switching technique to transfer voice messages in the form of discrete packets. Different packets from different users can be sent over the same network, thus reducing the bandwidth requirement.

\subsection{Steps Involved in making a VoIP Call}

The following steps take place at the transmission end in the following order:

$\begin{aligned} \text { i. } & \text { Signaling and media channel setup } \\ \text { ii. } & \text { Digitization of the analog voice signal } \\ \text { iii. } & \text { Encoding } \\ \text { iv. } & \text { Packetization } \\ \text { v. } & \text { Transmission of Packets over the Network }\end{aligned}$

On the receiving end, the reverse of the above said steps takes place:

$\begin{aligned} \text { i. } & \text { Receive the IP Packets } \\ \text { ii. } & \text { Decode the Packets } \\ \text { iii. } & \text { Convert the digital packets into analog signals }\end{aligned}$




\section{SESSION INITIATION PROTOCOL}

The Session Initiation Protocol is a text-based signaling communications protocol, which is used to creation, management and terminations of each session. It is responsible for smooth transmission of data packets over the network. It considers the request made by the user to make a call and then establishes connection between two or multiple users. When the call is complete, it destroys the session.

SIP can be used for two party (unicast) or multi party (multicast) sessions. It works in along with other application layer protocols that identify and carry the session media.

The SIP was developed by the Internet Engineering Task Force (IETF) and it supports RFC 3261. The protocol itself provides reliability and does not depend on TCP for reliability. Also, it depends on the Session Description Protocol (SDP) which is responsible for the negotiation for the codec identification.

\subsection{Services Provided by the SIP}

The Session Initiation Protocol provides various services to the users, some of which include the following:
(i) Locate User
(ii) Session Establishment
(iii) Session Setup Negotiation
(iv) Modify Session
(v) Teardown/End Session

\subsection{Components of SIP}

The SIP relies on a peer to peer (P2P) architecture which uses intelligent network elements for advanced call processing and call management functions. The various components of SIP are:

(i) User Agent - The actual user who wishes to make a call. The user agent itself has two more parts:
(a) User Agent Client (UAC)
(b) User Agent Server (UAS)

(ii)

Network Servers - There are basically three types of Servers involved in the protocol:
(a) Proxy Server
(b) Next Hop Server
(c) Redirect Server

\subsection{SIP Messages}

As we know that the SIP is a text based protocol which has syntax similar to another text based protocol Hyper Text Transfer Protocol (HTTP). The RFC 3261 defines the various types of messages that the SIP can support. The SIP messages fall widely under two categories, Requests and Responses. Some of the SIP supported messages are:

(a) REGISTER - Registers a user with a SIP server

(b) INVITE - Used to invite to participate in a Call session

(c) ACK - Acknowledge an INVITE request

(d) CANCEL - Cancel a pending request

(e) OPTIONS - Lists the information about the capabilities of the caller

(f) BYE - Terminates a connection

\section{VoIP ATTACKS}

There are different types of ways in which hackers can attack a VoIP call. Some of them are:

(a) Call Flooding - It is basically a form of DoS (Denial of Service) attack. This type of attack denies the services of the network to the user typically by flooding or overloading the network beyond it bandwidth.

(b) Phreaking - It is a type of service theft in which an attacker steals the service of the ISP (Internet Service Provider) while the service is being transmitted to a customer.

(c) Eavesdropping - This term is used to refer to an attack in which the attacker steals the personal messages or credentials of a user which the attacker is not intended to see. Through eavesdropping, the attacker can obtain useful personal information such as Usernames, Passwords, PIN's or any other confidential information.

(d) Phishing - In this type of attack the attacker uses some kind of software to make calls to customer with fake identities such as pretending to be a bank executive to steal personal information.

(e) Call Tampering - As the name suggests, this is an attack in which the attacker can tamper a VoIP call in progress. The quality of the call can thereby be harmed.

(f) Man-in-middle-attack - The man-in-middle attack can be set up simply by spoofing the MAC (Media Access Control) address of the SIP register. The attacker can masquerade as the calling party and thus hijack the call.

\subsection{VoIP Spoofing}

The practice of masquerading as a different caller rather than the actual caller is known as VoIP Spoofing or Caller ID Spoofing. Attackers use this technique to hide their identities thereby making them untraceable. In other words it is basically taking the identity of a host to make calls to another host without revealing the true identity of the host from where the call actually originates.

\subsection{Why Spoof Calls?}

The spoofing technique can be used for one of the following reasons:
(a) Obscuring the origin or packet
(b) Masquerading as a trusted host
(c) Obtaining personal information like bank account numbers, passwords and many more
(d) Hijacking of network traffic
(e) Hijacking a customer's network ID to use the network
(f) Directing Response to another host or system

\section{OVERVIEW OF ASTERISK SERVER}

Asterisk is basically software based Private Branch Exchange (PBX) which is designed to run only on a Linux system. It allows the user to connect to multiple telephones to make calls and to utilize other telephone services such as the PSTN and VoIP. Here, we are mainly concerned about the use of Asterisk to make VoIP calls. The Asterisk has its own extension languages and new functions can easily be created by the user. Asterisk supports many Voice over IP Protocols which includes the Session Initiation Protocol (SIP). The SIP uses the Asterisk server to make calls over the Internet. When the SIP originates a call, it sends it to the server with the address of the receiving party and the server then dispatches the packets which are eventually received by the receiver. Although there are various ways to secure an Asterisk server, but still there are loop holes. 


\section{PUBLIC KEY INFRASTRUCTURE AND PROPOSED SOLUTION}

The concept of Public Key Infrastructure is used to create, manage and revoke digital certificates. It is mostly used by customers with unsecured network. The certificate issued by the issuing authority marks the originality of the network. These kinds of certificates are known as Digital certificates. Every user using the PKI technology should have a registered identity. These identities are then stored in the form of Digital Certificates. The certificates issued by the Certificating authority are encrypted and thus any tampering with it can be easily detected. The certificates are encrypted through the concept of cryptography. Some of the commonly used encryption techniques are OpenPGP and S/MIME.

This proposed model tries to integrate the concepts of Public Key Infrastructure and the Asterisk Server which supports the Session Initiation Protocol of VoIP to make a much more secure Networking environment. The main task for securing the network using PKI concepts is by issuing a pair of Digital Certificates to the Asterisk Server as well as the Session Initiation Protocol. Once issued, any tampering with the network or the SIP would be easily detected by the Asterisk Server. When an attacker tries to spoof a call, he/she tries to interfere with the network address of the vulnerable user thereby changing the contents of the certificate. To proceed further, the attacker requires changing the original network address in the certificate by the network address he/she is using. This manipulation will be detected at the server during the dispatching of digital packets. The server will compare the copy of the certificate it has with the certificate it received from the host requesting to call. This concept will not only prevent us from receiving fake/spoofed call but the extension of this idea can also help us to identify the network address of the person trying to spoof the call. The idea would definitely make calling over the internet much more secure. When simulated, the server detects whenever a change in the certificate is made. It does not forward the call unless the certificates match. Thus, the simulation results advocates this idea of integrating the PKI concepts for making calls over the Internet.

\section{ADVANTAGES/DISADVANTAGES}

- The proposed solution will make the network more shielded and reliable and making/receiving calls over the internet would be more trustworthy.

- The concept of issuing certificate pairs to the SIP as well as Asterisk will be time consuming and more network bandwidth would be required in order to compare certificates every time a call is made.

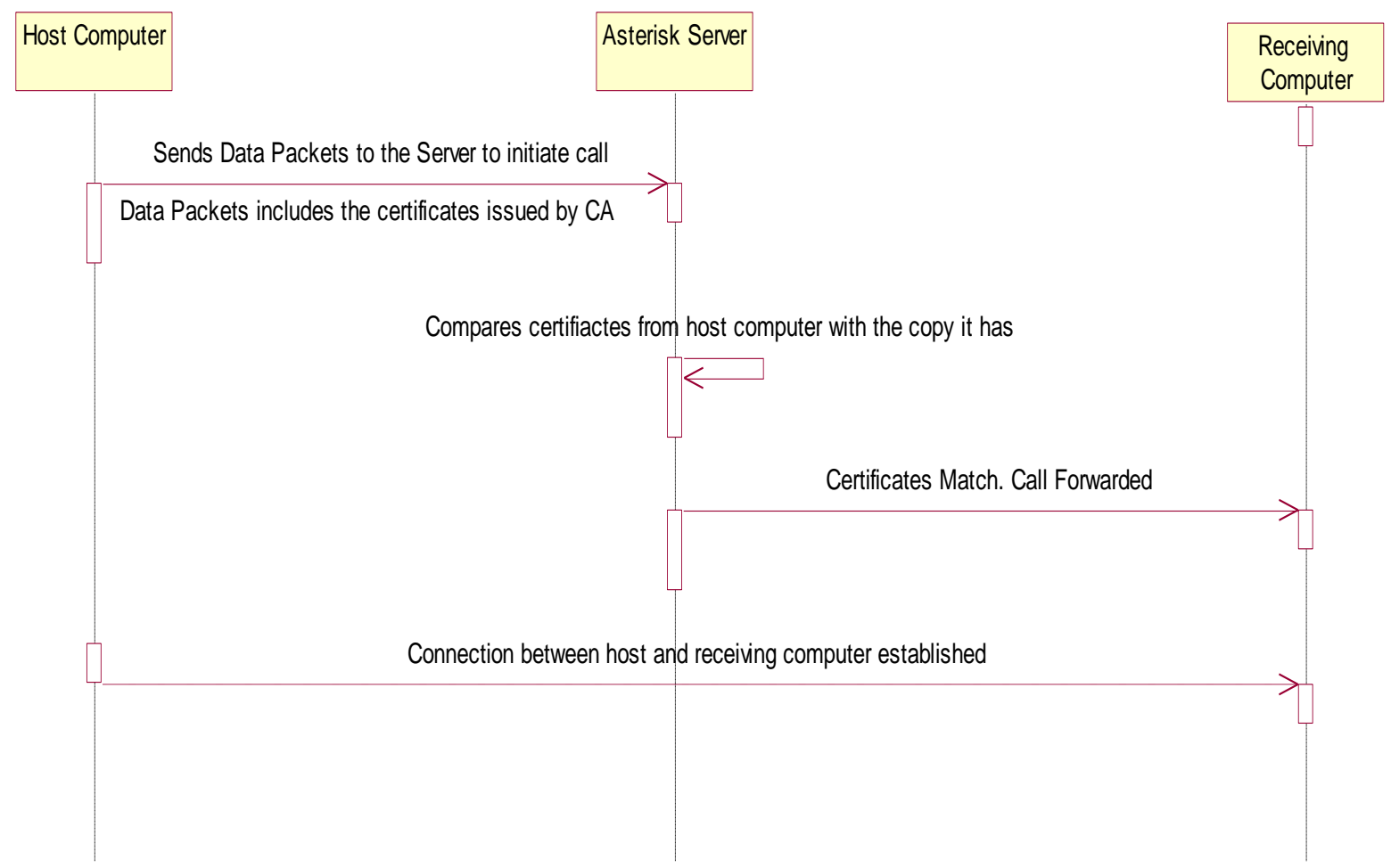

Figure. 1 Mechanism of VoIP Calling when Certificates Match 

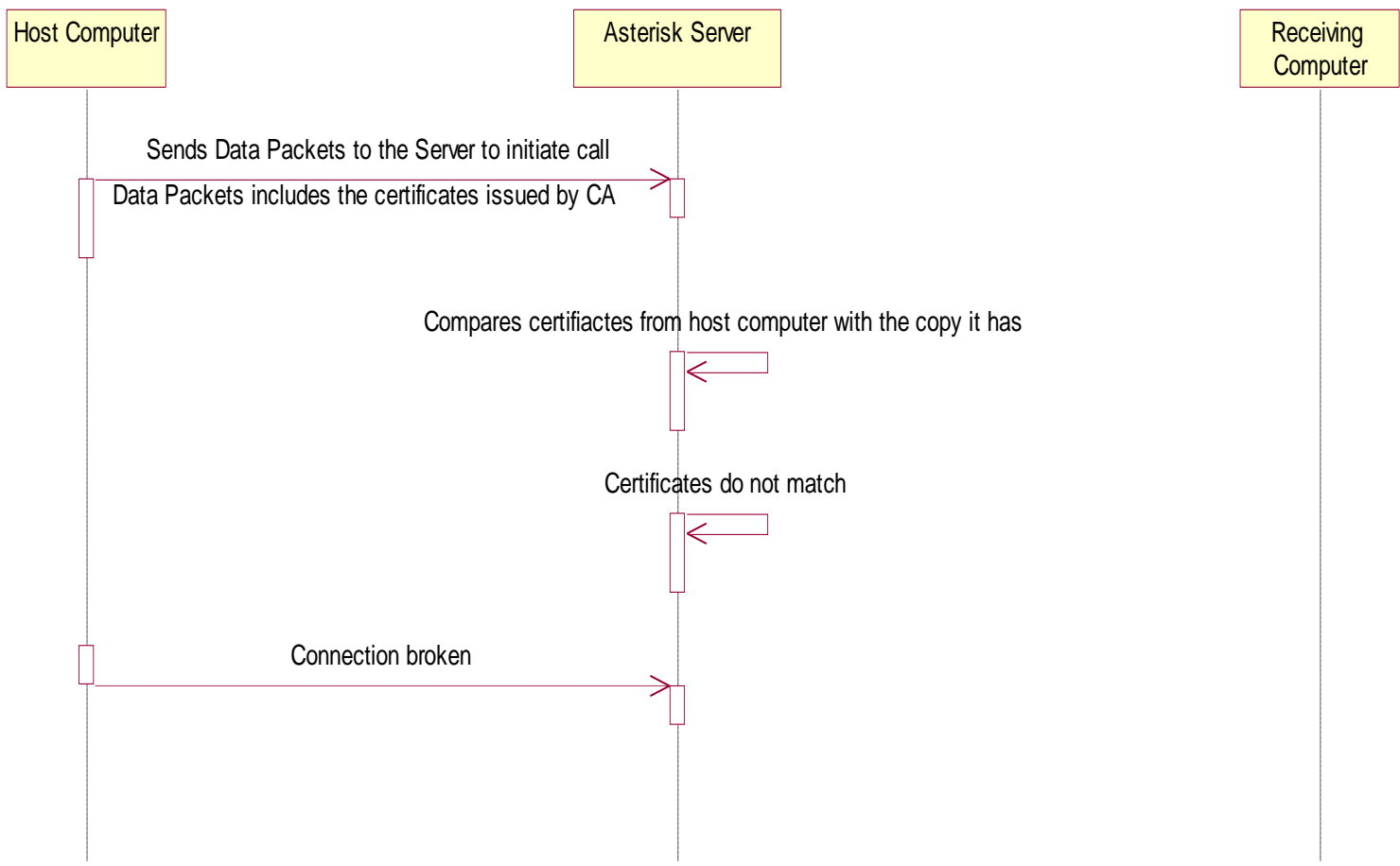

Figure. 2 Mechanism of VoIP calling when Certificates do not match

\section{CONCLUSION}

The paper mainly focuses on the security issues of VoIP over Internet Protocol. From the simulation results, it can be concluded that VoIP is a very attractive way to communicate owing to is low cost and rich feature set. In spite of all the appealing features that VoIP offers, it is open to vulnerabilities and threats. First, this paper talks about the basic concepts of making a call over the internet. Next it analyzes in detail, the Session Initiation Protocol (SIP) and the Asterisk Server which forms the background of making calls by using the Internet Protocol. Also, it is understood that it is fairly easy to attack a voice call made over the internet. Through the observations, it can be inferred that the attacks can be minimized by simply certifying the protocol as well as the server by a trusted certificating authority. Although, it is time consuming to certify the network address of each user, but once issued it can rectify the security issues. The main reason that VoIP technology has not yet replaced the traditional PSTN is the security issues. Therefore, it becomes pretty inevitable to resolve the security issues of VoIP so that we can shift to a more reliable and an attractive source of voice communication.

\section{REFERENCES}

[1] Rakesh Arora "Voice Over IP Protocols and Standards"

[2] Sanjay Kumar Sonkar, Rahul Singh, Ritu Chauhan, Ajay Pal Singh on "Security of Voice over Internet Protocol from Spoofing attacks", International Journal of Advanced Research in Computer and Communication Engineering Volume 1, Issue 3, May 2012.
[3] Ge Zhang, "Secure SIP Signaling Service for VoIP applications Performance-Related Attacks and Prevention".

[4] Angelos D. Keromytis, "A comprehensive survey on Voice over IP Security and Research"

[5] H. Abdelnur, T. Avanesov, M. Rusinowitch, and R. State, "Abusing SIP Authentication," in Proceedings of the 4th International Conference on Information Assurance and Security (ISIAS), September 2008.

[6] Seedorf, J., "SIP Security: Status Quo and Future Issues", Talk presented at 23rd Chaos Communication Congress, 2006.

[7] A. Kurmus and J.-F.Garet, "Studying and Experimenting with threats against Voice over IP Systems," Technical Representation Masters Thesis EURECOM 2009.

[8] Mohammad Masudur Rahman, Nafish Sarwar Islam, "VoIP Implementation using Asterisk PBX", IOSR Journal of Business and Management (IOSR-JBM), Volume 15, Issue 6, January 2014.

[9] William Stallings, "Cryptography and Network Security", USA.

[10] Riddhi Patel, Pushkar Jha, Aditya K. Sinha, "Analysis of SIP Authentication in VoIP", International Journal of Engineering Research and Technology (IJERT), ISSN: 2278-0181, Volume 2, Issue 4, April 2013. 